\title{
Learning Finite State Machines With Self-Clustering Recurrent Networks
}

\author{
Zheng Zeng \\ Rodney M. Goodman \\ Department of Electrical Engineering, 116-81, \\ California Institute of Technology, Pasadena, CA 91125 USA \\ Padhraic Smyth \\ Jet Propulsion Laboratory, 238-420, California Institute of Technology \\ Pasadena, CA 91109 USA
}

Recent work has shown that recurrent neural networks have the ability to learn finite state automata from examples. In particular, networks using second-order units have been successful at this task. In studying the performance and learning behavior of such networks we have found that the second-order network model attempts to form clusters in activation space as its internal representation of states. However, these learned states become unstable as longer and longer test input strings are presented to the network. In essence, the network "forgets" where the individual states are in activation space. In this paper we propose a new method to force such a network to learn stable states by introducing discretization into the network and using a pseudo-gradient learning rule to perform training. The essence of the learning rule is that in doing gradient descent, it makes use of the gradient of a sigmoid function as a heuristic hint in place of that of the hard-limiting function, while still using the discretized value in the feedback update path. The new structure uses isolated points in activation space instead of vague clusters as its internal representation of states. It is shown to have similar capabilities in learning finite state automata as the original network, but without the instability problem. The proposed pseudo-gradient learning rule may also be used as a basis for training other types of networks that have hard-limiting threshold activation functions.

\section{Introduction}

Theoretical aspects of grammatical inference have been studied extensively in the past (Angluin 1972, 1978; Gold 1972, 1978). A variety of direct search algorithms have been proposed for learning grammars from positive and negative examples (strings) (Angluin and Smith 1983; Fu 
1982; Muggleton 1990; Tomita 1982). More recently recurrent neural networks have been investigated as an alternative method for learning simple grammars (Cleeremans et al. 1989; Elman 1990, 1991; Fahlman 1990; Giles et al. 1990, 1992; Jordan 1986; Pollack 1991; Rumelhart et al. 1986; Williams and Zipser 1989). A variety of network architectures and learning rules have been proposed. All have shown the capability of recurrent networks to learn different types of simple grammars from examples.

In this paper we restrict the focus to studying a recurrent network's behavior in learning regular grammars, which are the simplest type of grammar in the Chomsky hierarchy, and have a one-to-one correspondence to finite state machines (Hopcroft and Ullman 1979). A regular language can be defined as the language accepted by its corresponding finite state acceptor: $\left\langle\Sigma, T, t_{0}, \delta, F\right\rangle$, where

- $\Sigma$ is the input alphabet.

- $T$ is a finite nonempty set of states.

- $t_{0}$ is the start (or initial) state, an element of $T$.

- $\delta$ is the state transition function; $\delta: T \times \Sigma \rightarrow T$.

- $F$ is the set of final (or accepting) states, a (possibly empty) subset of $T$.

The purpose of the study is to obtain a better understanding of recurrent neural networks, their behavior in learning, and their internal representations, which in turn may give us more insight into their capability for fulfilling other more complicated tasks.

Giles et al. $(1990,1992)$ have proposed a "second-order" recurrent network structure to learn regular languages. Henceforth, all references to second-order recurrent networks imply the network structure described in Giles et al. $(1990,1992)$. Our independent experiments have confirmed their results that second-order nets can learn various grammars well. In addition, we found that this structure learns these grammars more easily than the simple recurrent network structure (or the Elman structure) (Elman 1991) which does not use second-order units. However, a stability problem emerges with trained networks as longer and longer input strings are presented [similar behavior in recurrent networks has been found in different contexts (Pollack 1991; Servan-Schreiber et al. 1991)]. In our experiments, the problem appears in 14 out of 15 of the trained networks on nontrivial machines for long strings. A string can be misclassified as early as when its length is only $30 \%$ longer than the maximum training length in some of the experiments. The stability problem led us to look deeper into the internal representation of states in such a network and the following interesting behavior was observed: during learning, the network attempts to form clusters in hidden unit space as its representation of states. This behavior occurred in all the learning experiments we performed. Once formed, the clusters are stable for short strings, i.e., 
strings with lengths not much longer than the maximum length of training strings. However, in 14 out of 15 learned networks, when sufficiently long strings are presented for testing, the clusters (states) start to merge and ultimately become indistinguishable. (Details of these experiments will be explained in Section 2.) To solve this problem we propose a discretized combined network structure, as well as a pseudo-gradient learning method, which can be shown to successfully learn stable state representations. In the proposed network, instead of clusters, the states of the network are actually isolated points in hidden unit activation space.

\section{The "Unstable State" Behavior of a Learned Second-Order Net}

We found that the second-order network can be represented as two separate networks controlled by a gating switch (Fig. 1) as follows: the network consists of two first-order networks with shared hidden units. The common hidden unit values are copied back to both net0 and net1 after each time step, and the input stream acts like a switching control to enable or disable one of the two nets. For example, when the current input is 0 , neto is enabled while net1 is disabled. The hidden unit values are then decided by the hidden unit values from the previous time step weighted by the weights in neto. The hidden unit activation function is the standard sigmoid function, $f(x)=1 /\left(1+e^{-x}\right)$. Note that this representation of a second-order network, as two networks with a gating function, provides insight into the nature of second-order nets, i.e., clearly they have greater representational power than a single simple recurrent network, given the same number of hidden units. This structure was used in our initial experiments.

We use $S_{i}^{t}$ to denote the activation value of hidden unit number $i$ at time step $t$. $w_{i j}^{n}$ is the weight from layer 1 node $j$ to layer 2 node $i$ in netn. $n=0$ or 1 in the case of binary inputs. Hidden node $S_{0}^{t}$ is chosen to be a special indicator node, whose activation should be close to 1 at the end of a legal string, or close to 0 otherwise. At time $t=0$, initialize $S_{0}^{0}$ to be 1 and all other $S_{i}^{0} \mathrm{~s}$ to be 0 , i.e., assume that the null string is a legal string. The network weights are initialized randomly with a uniform distribution between -1 and 1 .

In the experiments described here we used the following grammars:

- Tomita grammars (Tomita 1982):

○\#1-1*.

- \#4-any string not containing " 000 " as a substring.

0 \#5-even number of $0 \mathrm{~s}$ and even number of $1 \mathrm{~s}$.

○ \#7-0*1*0*1*.

- Simple vending machine (Carroll and Long 1989): The machine takes in three types of coins: nickel, dime, and quarter. Starting 


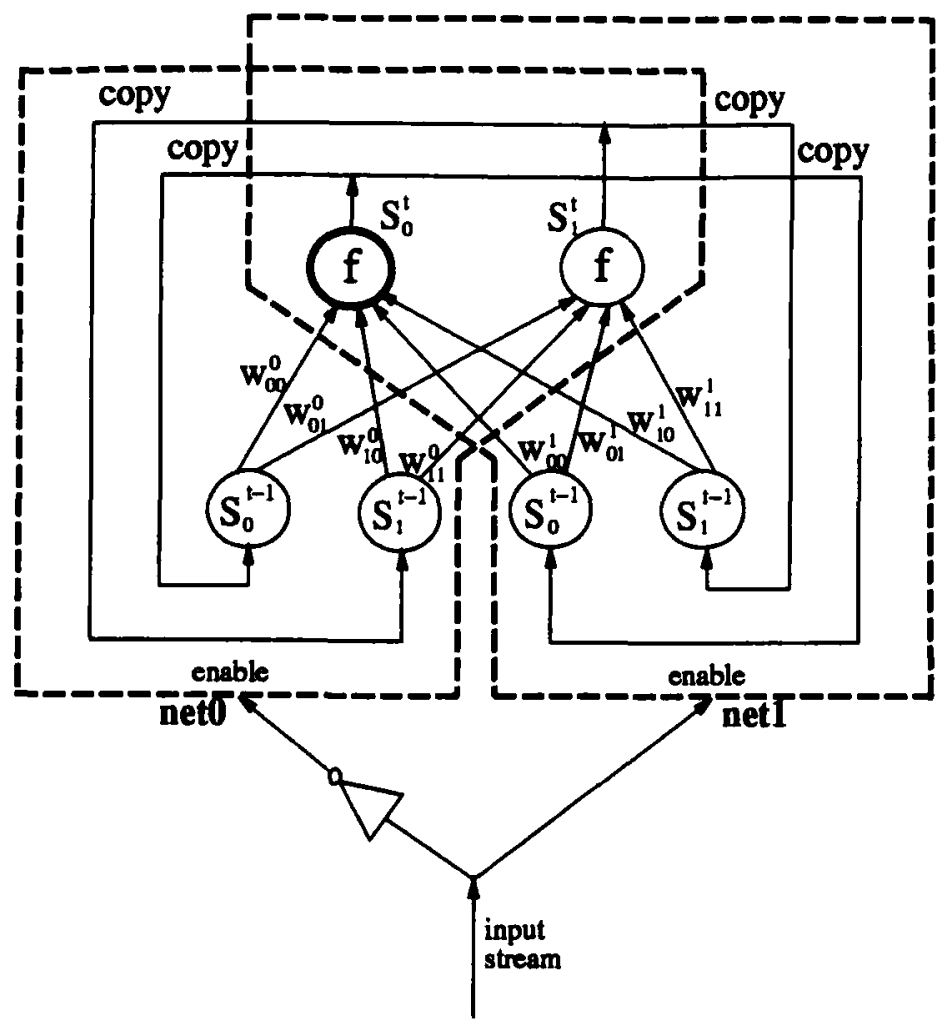

Figure 1: Equivalent First-order structure of second-order network.

from empty, a string of coins is entered into the machine. The machine "accepts," i.e., a candy bar may be selected, only if the total amount of money entered exceeds 30 cents.

A training set consists of randomly chosen variable length strings with length uniformly distributed between 1 and $L_{\max }$, where $L_{\max }$ is the maximum training string length. Each string is marked as "legal" or "illegal" according to the underlying grammar. The learning procedure is a gradient descent method in weight space [similar to that proposed by Williams and Zipser (1989)] to minimize the error at the indicator node for each training string (Giles et al. 1992).

In a manner different from that described in Giles et al. (1992), we present the whole training set (which consists of 100 to 300 strings with $L_{\max }$ in the range of 10 to 20), all at once to the network for learning, 


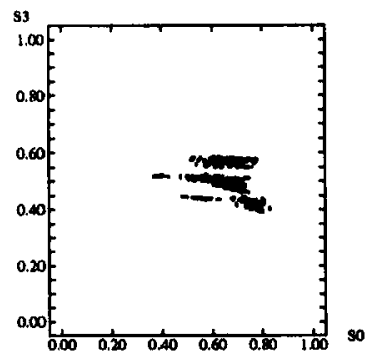

(a)

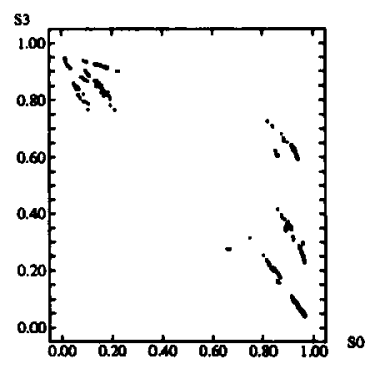

(d)

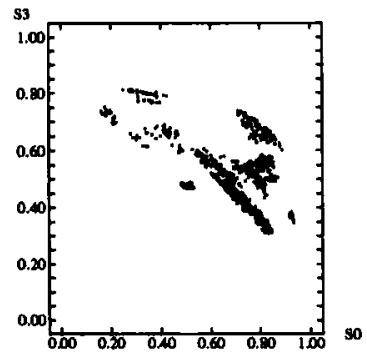

(b)

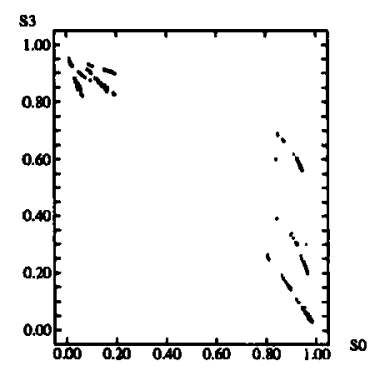

(e)

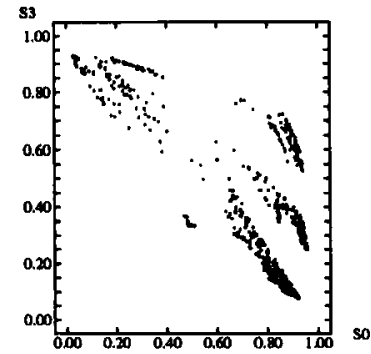

(c)

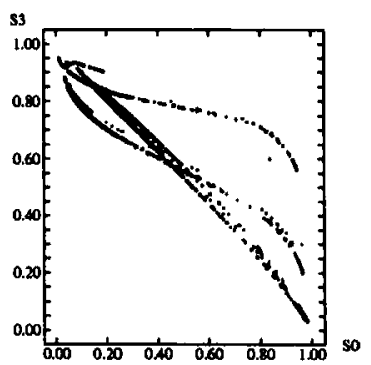

(f)

Figure 2: Hidden unit activation plot $S_{0}-S_{3}$ in learning Tomita grammar \#4. ( $S_{0}$ is the $x$ axis.) (a)-(e) are plots of all activations on the training data set. (a) During 1st epoch of training. (b) During 16th epoch of training. (c) During 21st epoch of training. (d) During 31st epoch of training. (e) After 52 epochs, training succeeds, weights are fixed. (f) After training, when tested on a set of maximum length 50 .

instead of presenting a portion of it in the beginning and gradually augmenting it as training proceeds. Also, we did not add any end symbol to the alphabet as in Giles et al. (1992). We found that the network can successfully learn the machines ( $2-10$ states) we tested on, with a small number of hidden units (4-5) and less than 500 epochs, agreeing with the results described in Giles et al. (1992).

To examine how the network forms its internal representation of states, we recorded the hidden unit activations at every time step of every training string in different training epochs. As a typical example, shown in Figure 2a-e, are the $S_{0}-S_{3}$ activation-space records of the learning process of a 4-hidden-unit network. The underlying grammar 
was Tomita \#4, and the training set consisted of 100 random strings with $L_{\max }=15$. Note that here the dimension $S_{0}$ is chosen because of it being the important "indicator node," and $S_{3}$ is chosen arbitrarily. The observations that follow can be made from any of the 2-D plots from any run in learning any of the grammars in the experiments. Each point corresponds to the activation pattern of a certain time step in a certain string. Each plot contains the activation points of all time steps for all training strings in a certain training epoch as described in the caption. The following behavior can be observed:

1. As learning takes place, the activation points seem to be pulled in several different directions, and distinct clusters gradually appear (Fig. 2a-e).

2. After learning is complete, i.e., when the error on each of the training strings is below a certain tolerance level, the activation points form distinct clusters, which consist of segments of curves (Fig. 2e).

3. Note in particular that there exists a clear gap between the clusters in the $S_{0}$ (indicator) dimension, which means that the network is making unambiguous decisions for all the training strings and each of their prefix strings (Fig. $2 \mathrm{e}$ ).

4. When given a string, the activation point of the network jumps from cluster to cluster as input bits are read in one by one. Hence, the behavior of the network looks just like a state machine's behavior.

It is clear that the network attempts to form clusters in activation space as its own representation of states and is successful in doing so. Motivated by these observations, we applied the $k$-means clustering algorithm to the activation record in activation space of the trained network to extract the states [instead of simply dividing up the space evenly as in Giles et al. (1992)]. In choosing the parameter $k$, we found that if $k$ was chosen too small, the extracted machine sometimes could not classify all the training strings correctly, while a large $k$ always guaranteed perfect performance on training data. Hence, $k$ was chosen to be a large number, for example, 20.

The initial seeds were chosen randomly. We then defined each cluster found by the $k$-means algorithm to be a "state" of the network and used the center of each cluster as a representative of the state. The transition rules for the resulting state machine are calculated by setting the $S_{i}^{t-1}$ nodes equal to a cluster center, then applying an input bit $(0$ or 1 in binary alphabet case), and calculating the value of the $S_{i}^{t}$ nodes. The transition from the current state given the input bit is then to the state that has a center closest in Euclidean distance to the obtained $S_{i}^{t}$ values. In all our experiments, the resulting machines were several states larger than the correct underlying minimal machines. Moore's state machine reduction algorithm was then applied to the originally extracted machine 
to get an equivalent minimal machine which accepts the same language but with the fewest possible number of states. Similar to the results in Giles et al. (1992), we were able to extract machines that are equivalent to the minimal machines corresponding to the underlying grammars from which the data was generated.

These trained networks perform well in classifying unseen short strings (not much longer than $L_{\max }$ ). However, as longer and longer strings are presented to the network, the percentage of strings correctly classified drops substantially. Shown in Figure $2 \mathrm{f}$ is the recorded activation points for $S_{0}-S_{3}$ of the same trained network from Figure $2 \mathrm{e}$ when long strings are presented. The original net was trained on 100 strings with $L_{\max }=15$, whereas the maximum length of the test strings in Figure $2 \mathrm{e}$ was 50 . Activation points at all time steps for all test strings are shown.

Several observations can be made from Figure 2e:

1. The well-separated clusters formed during training begin to merge together for longer and longer strings and eventually become indistinguishable. These points in the center of Figure $2 \mathrm{e}$ correspond to activations at time steps longer than $L_{\max }=15$.

2. The gap in the $S_{0}$ dimension disappears, which means that the network could not make hard decisions on long strings.

3. The activation points of a string stay in the original clusters for short strings and start to diverge from them when strings become longer and longer. The diverging trajectories of the points form curves with sigmoidal shape.

Similar behavior was observed for 14 out of 15 of the networks successfully trained on different machines, excluding the vending machine model. Some of the networks started to misclassify as early as when the input strings were only $30 \%$ longer than $L_{\max }$. Each of these 14 trained networks made classification errors on randomly generated test sets with maximum string length no longer than $5 L_{\max }$. The remaining one network was able to maintain a stable representation of states for very long strings (up to length 1000). Note that the vending machine was excluded because it is a trivial case for long strings, i.e., all the long strings are legal strings so there is no need to distinguish between them. This is not the case for the other machines.

\section{A Network That Can Form Stable States}

From the above experiments it is clear that even though the network is successful in forming clusters as its state representation during training, it often has difficulty in creating stable clusters, i.e., to form clusters such that the activation points for long strings converge to certain centers of 
each cluster, instead of diverging as observed in our experiments. The problem can be considered as inherent to the structure of the network where it uses analog values to represent states, while the states in the underlying state machine are actually discrete. One intuitive suggestion to fix the problem is to replace the analog sigmoid activation function in the hidden units with a threshold function:

$$
D(x)= \begin{cases}1.0 & \text { if } x \geq 0.5 \\ 0.0 & \text { if } x<0.5\end{cases}
$$

In this manner, once the network is trained, its representation of states (i.e., activation pattern of hidden units) will be stable and the activation points will not diverge from these state representations once they are formed. However, there is no known method to train such a network, since one cannot take the gradient of such activation functions.

An alternative approach would be to train the original second-order network as described earlier, but to add the discretization function $D(x)$ on the copy back links during testing. The problem with this method is that one does not know a priori where the formed clusters from training will be. Hence, one does not have good discretization values to threshold the analog values in order for the discretized activations to be reset to a cluster center. Experimental results have confirmed this prediction. For example, after adding the discretization, the modified network cannot even correctly classify the training set that it has successfully learned in training. As in the previous example, after training and without the discretization, the network's classification rate on the training set was $100 \%$, while with the discretization added, the rate became $85 \%$. For test sets of longer strings, the rates with discretization were even worse.

We propose that the discretization be included in both training and testing in the following manner: Figure 3 shows the structure of the network with discretization added.

From the formulas below, one can clearly see that in operational mode, that is, when testing, the network is equivalent to a network with discretization only:

$$
\begin{array}{rlrl}
h_{i}^{t} & =f\left(\sum_{j} w_{i j}^{x^{t}} S_{j}^{t-1}\right), & \forall i, t, & \text { where } D(x)= \begin{cases}0.8 & \text { if } x \geq 0.5 \\
0.2 & \text { if } x<0.5,\end{cases} \\
S_{i}^{t} & =D\left(h_{i}^{t}\right), & \\
\Rightarrow S_{i}^{t} & =D\left[f\left(\sum_{j} w_{i j}^{x^{t}} S_{j}^{t-1}\right)\right] & & \text { where } D_{0}(x)= \begin{cases}0.8 & \text { if } x \geq 0.0 \\
0.2 & \text { if } x<0.0 .\end{cases}
\end{array}
$$

(Here $x^{t}$ is the input bit at time step $t$. We use $h_{i}^{t}$ to denote the analog value of hidden unit $i$ at time step $t$, and $S_{i}^{t}$ the discretized value of hidden unit $i$ at time step $t$.) 


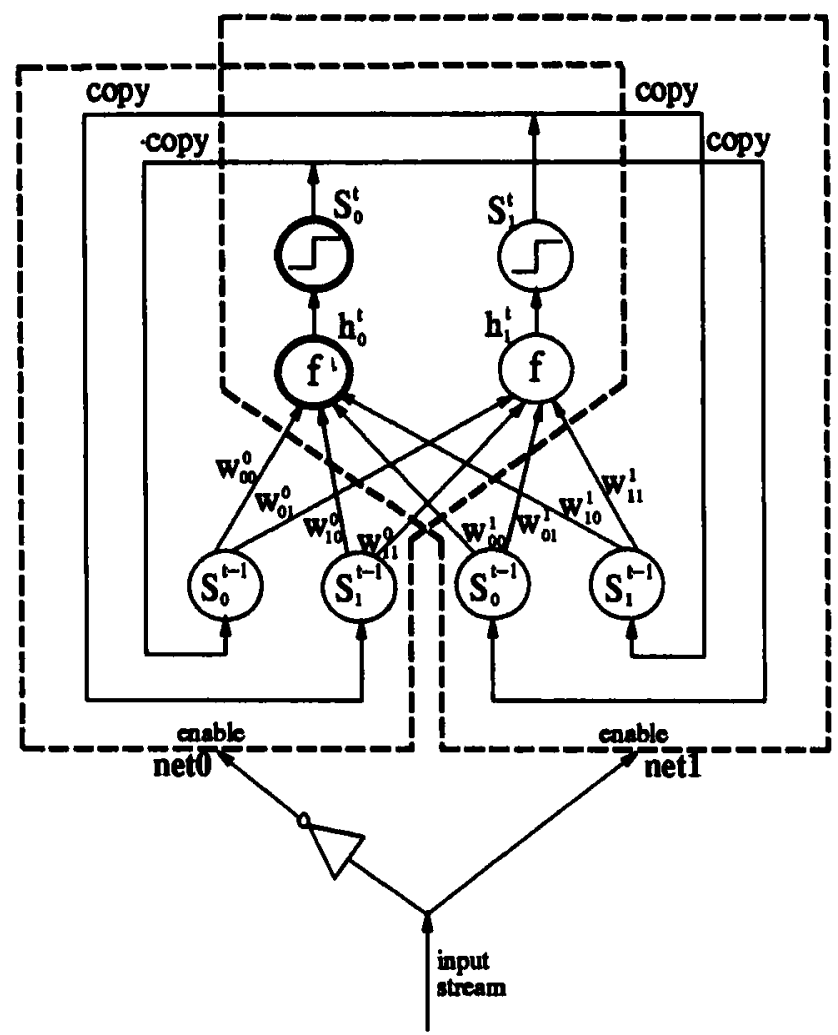

Figure 3: A combined network with discretizations.

Hence, the sigmoid nodes can be eliminated in testing to simplify computation.

During training, however, the gradient of the soft sigmoid function is made use of in a pseudo-gradient method for updating the weights. The next section explains the method in more detail.

By adding these discretizations into the network, one might argue that the capacity of the net is greatly reduced, since each node can now take on only two distinct values, as opposed to infinitely many values (at least in theory) in the case of the undiscretized networks. However, in the case of learning discrete state machines, the argument depends on the definition of the capacity of the analog network. In our experiments, 14 of 15 of the learned networks have unstable behavior for nontrivial long strings, so one can say that the capabilities of such networks to distinguish different states may start high, but deteriorate over time, and would eventually become zero. 


\section{The Pseudo-Gradient Learning Method}

During training, at the end of each string: $x^{0}, x^{1}, \ldots, x^{L}$, the mean squared error is calculated as follows (note that $L$ is the string length, $h_{0}^{L}$ is the analog indicator value at the end of the string):

$$
E=\frac{1}{2}\left(h_{0}^{L}-T\right)^{2}
$$

where

$$
T=\text { target }= \begin{cases}1 & \text { if "legal" } \\ 0 & \text { if "illegal". }\end{cases}
$$

Update $w_{i j}^{n}$, the weight from node $j$ to node $i$ in netn, at the end of each string presentation:

$$
\begin{aligned}
& w_{i j}^{n}=w_{i j}^{n}-\alpha \frac{\widetilde{\partial E}}{\partial w_{i j}^{n}}, \quad \forall n, i, j, \\
& \frac{\widetilde{\partial E}}{\partial w_{i j}^{n}}=\left(h_{0}^{L}-T\right) \frac{{\widetilde{\partial h_{0}}}_{0}}{\partial w_{i j}^{n}}, \quad \forall n, i, j
\end{aligned}
$$

where $\tilde{\partial} / \partial w_{i j}^{n}$ is what we call the "pseudo-gradient" with respect to $w_{i j}^{n}$.

To get the pseudo-gradient ${\widetilde{\partial h_{0}}}_{0}^{L} / \partial w_{i j}^{n}$, pseudo-gradients ${\widetilde{\partial h_{k}}}_{k}^{t} / \partial w_{i j}^{n}$ for all $t, k$ need to be calculated forward in time at each time step:

$$
\frac{{\widetilde{\partial h_{k}}}_{k}^{t}}{\partial w_{i j}^{n}}=f^{\prime} \cdot\left(\sum_{l} w_{k l}^{x^{\prime}} \frac{{\widetilde{\partial h_{l}}}_{\partial-1}^{\partial w_{i j}^{n}}}{{ }^{t-1}} \delta_{k i} \delta_{n x^{\prime}} S_{j}^{t-1}\right), \quad \forall i, j, n, k, t
$$

(Initially, set: $\quad \widetilde{\partial h}_{k}^{0} / \partial w_{i j}^{n}=0, \quad \forall i, j, n, k$ )

As can be seen clearly, in carrying out the chain rule for the gradient we replace the real gradient $\partial S_{l}^{t-1} / \partial w_{i j}^{n}$, which is zero almost everywhere, by the pseudo-gradient $\widetilde{\partial h_{l}^{t-1}} / \partial w_{i j}^{n}$. The justification of the use of the pseudo-gradient is as follows: suppose we are standing on one side of the hard threshold function $S(x)$, at point $x_{0}>0$, and we wish to go downhill. The real gradient of $S(x)$ would not give us any information, since it is zero at $x_{0}$. If instead we look at the gradient of the function $f(x)$, which is positive at $x_{0}$ and increases as $x_{0} \rightarrow 0$, it tells us that the downhill direction is to decrease $x_{0}$, which is also the case in $S(x)$. In addition, the magnitude of the gradient tells us how close we are to a step down in $S(x)$. Therefore, we can use that gradient as a heuristic hint as to which direction (and how close) a step down would be. This heuristic hint is what we used as the pseudo-gradient in our gradient update calculation. 


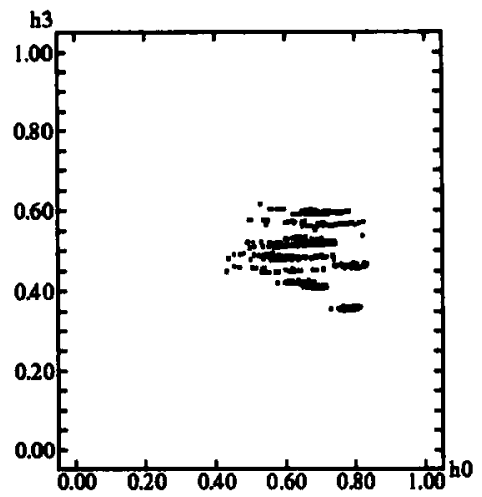

(a)

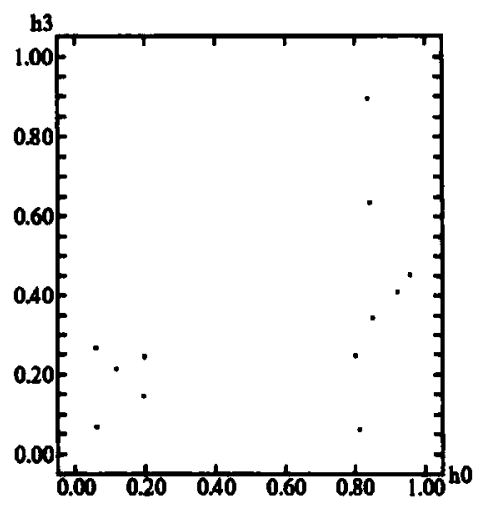

(c)

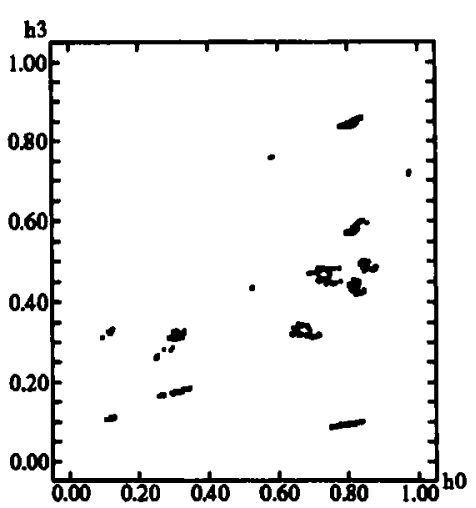

(b)

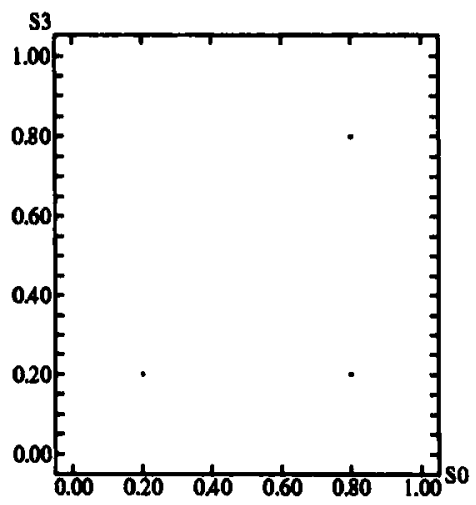

(d)

Figure 4: Discretized network learning Tomita grammar \# 4. (a) $h_{0}-h_{3}$ during 1 st epoch of training. (b) $h_{0}-h_{3}$ during 15th epoch of training. (c) $h_{0}-h_{3}$ after 27 epochs when training succeeds, weights are fixed. (d) $S_{0}-S_{3}$, the discretized copy of $h_{0}-h_{3}$ in (c).

\section{Experimental Results}

Shown in Figure $4 \mathrm{a}-\mathrm{c}$ are the $h_{0}-h_{3}$ activation-space records of the learning process of a discretized network ( $h$ values are the undiscretized values 
from the sigmoids). The underlying grammar is again the Tomita Grammar \#4. The parameters of the network and the training set are the same as in the previous case. Again, any of the other 2-D plots from any run in learning any of the grammar in the experiments could have been used here.

Figure $4 \mathrm{c}$ is the final result after learning, where the weights are fixed. Notice that there are only a finite number of points in the final plot in the analog activation $h$-space due to the discretization. Figure $4 \mathrm{~d}$ shows the discretized value plot in $S_{0}-S_{3}$, where only three points can be seen. Each point in the discretized activation $S$-space is automatically defined as a distinct state, no point is shared by any of the states. The transition rules are calculated as before, and an internal state machine in the network is thus constructed. In this manner, the network performs self-clustering. For this example, six points are found in $S$-space, so a six-state-machine is constructed as shown in Figure 5a. Not surprisingly this machine reduces by Moore's algorithm to a minimum machine with four states, which is exactly the Tomita Grammar \#4 (Fig. 5b). Similar results were observed for all the other grammars in the experiments.

There are several advantages in introducing discretization into the network:

1. Once the network has successfully learned the state machine from the training set, its internal states are stable. The network will always classify input strings correctly, independent of the lengths of these strings.

2. No clustering is needed to extract out the state machine, since instead of using vague clusters as its states, the network has formed distinct, isolated points as states. Each point in activation space is a distinct state. The network behaves exactly like a state machine.

3. Experimental results show that the size of the state machines extracted out in this approach, which need not be decided manually (no need to choose $k$ for $k$-means) as in the previous undiscretized case, is much smaller than found previously by the clustering method.

It should be noted that convergence has a different meaning in the case of training discrete networks as opposed to the case of training analog networks. In the analog networks' case, learning is considered to have converged when the error for each sample is below a certain errortolerance level. In the case of discrete networks, however, learning is stopped and considered to have converged only when zero error is obtained on all samples in the training set. In the experiments reported in this paper the analog tolerance level was set to 0.2 . The discretized networks took on average $30 \%$ longer to train in terms of learning epochs compared to the analog networks for this specific error tolerance level. 


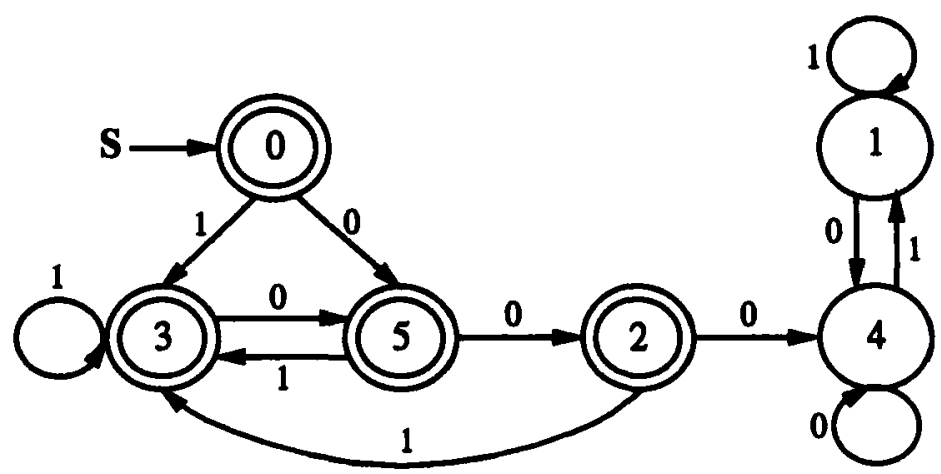

(a)

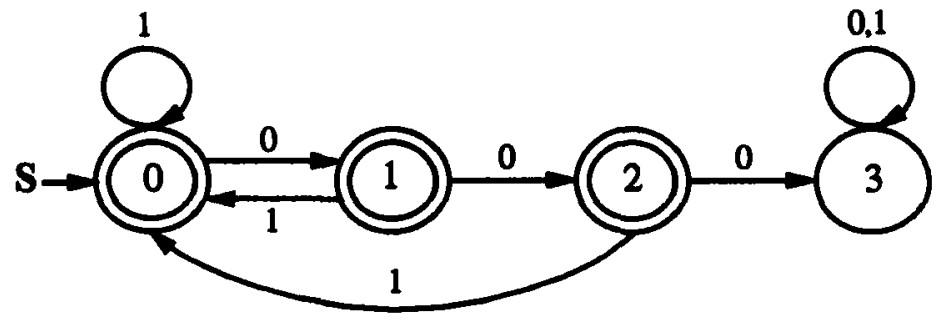

(b)

Figure 5: Extracted state machine from the discretized network after learning Tomita grammar \#4 (double circle means "accept" state, single circle means "reject" state). (a) Six-state machine extracted directly from the discrete activation space. (b) Equivalent minimal machine of (a).

\section{Conclusion}

In this paper we explored the formation of clusters in hidden unit activation space as an internal state representation for second-order recurrent networks that learn regular grammars.

These states formed by such a network during learning are not a stable representation, i.e., when long strings are seen by the network the states merge into each other and eventually become indistinguishable.

We suggested introducing hard-limiting threshold discretization into the network and presented a pseudo-gradient learning method to train such a network. The method is heuristically plausible and experimental results show that the network has similar capabilities in learning finite 
state machines as the original second-order network, but is stable regardless of string length since the internal representation of states in this network consists of isolated points in activation space.

The proposed pseudogradient learning method suggests a general approach for training networks with threshold activation functions.

\section{Acknowledgments}

The research described in this paper was supported in part by ONR and ARPA under Grants AFOSR-90-0199 and N00014-92-J-1860. In addition this work was carried out in part by the Jet Propulsion Laboratories, California Institute of Technology, under a contract with the National Aeronautics and Space Administration.

\section{References}

Angluin, D. 1972. Inference of reversible languages. J. Assoc. Comput. Machin. 29(3), 741-765.

Angluin, D. 1978. On the complexity of minimum inference of regular sets. Inform. Control 39, 337-350.

Angluin, D., and Smith, C. H. 1983. Inductive inference: theory and methods. ACM Computing Survey, 15(3), 237.

Carroll, J., and Long, D. 1989. Theory of Finite Automata. Prentice Hall, Englewood Cliffs, NJ.

Cleeremans, A., Servan-Schreiber, D., and McClelland, J. L. 1989. Finite state automata and simple recurrent networks. Neural Comp. 1, 372-381.

Elman, J. L. 1990. Finding structure in time. Cog. Sci. 14, 179-211.

Elman, J. L. 1991. Distributed representations, simple recurrent networks, and grammatical structure. Machine Learn. 7(2/3), 195-225.

Fahlman, S. E. 1990. The recurrent cascade-correlation architecture. In Advances in Neural Information Processing Systems, pp. 190-196.

Fu, K. S. 1982. Syntactic Pattern Recognition and Applications. Prentice Hall, Englewood Cliffs, NJ.

Giles, C. L., Sun, G. Z., Chen, H. H., Lee, Y. C., and Chen, D. 1990. Higher order recurrent networks and grammatical inference. In Advances in Neural Information Processing Systems, pp. 380-387.

Giles, C. L., Miller, C. B., Chen, D., Chen, H. H., Sun, G. Z., and Lee, Y. C. 1992. Second-order recurrent neural networks. Neural Comp. 4(3), 393-405.

Gold, E. M. 1972. System identification via state characterization. Automatica 8, 621-636.

Gold, E. M. 1978. Complexity of automaton identification from given data. Information and Control 37, 302-320.

Hopcroft, J. E., and Ullman, J. D. 1979. Introduction to Automata Theory, Languages and Computation. Addison-Wesley, Reading MA. 
Jordan, M. I. 1986. Serial order: A parallel distributed processing approach. Tech. Rep. No. 8604, San Diego: University of California, Institute for Cognitive Science.

Kudo, M., and Shimbo, M. 1988. Efficient regular grammatical inference techniques by the use of partial similarities and their logical relationships. Pattern Recog. 21(4), 401-409.

Muggleton, S. 1990. Grammatical Induction Theory. Addison-Wesley, Turing Institute Press, Reading, MA.

Pollack, J. B. 1991. The induction of dynamical recognizers. Machine Learn. 7(2/3), 227-252.

Rumelhart, D. E., McClelland, J. L., and the PDP Research Group. 1986. In Parallel Distributed Processing, pp. 354-361. The MIT Press.

Servan-Schreiber, D., Cleeremans, A., and McClelland, J. L. 1991. Graded state machines: The representation of temporal contingencies in simple recurrent networks. Machine Learn. 7(2/3), 161-193.

Tomita, M. 1982. Dynamic construction of finite-state automata from examples using hill climbing. In Proceedings of the Fourth Annual Cognitive Science Conference, p. 105.

Williams, R. J., and Zipser, D. 1989. A learning algorithm for continually running fully recurrent neural networks. Neural Comp. 1(2), 270-280.

Received 15 June 1992; accepted 8 March 1993. 